\title{
Sarcopenia is a reliable predictor of outcomes following radical cystectomy for bladder cancer
}

\author{
Matthew G. Kaag, Jay D. Raman \\ Department of Surgery (Urology), Penn State Health, Hershey, PA, USA \\ Correspondence to: Matthew G. Kaag. Department of Surgery (Urology), Penn State Health, Hershey, PA 17033, USA. Email: mkaag@pennstatehealth.psu.edu. \\ Provenance: This is an Invited Editorial commissioned by Section Editor Xiao Li (Department of Urology, Jiangsu Cancer Hospital \& Jiangsu \\ Institute of Cancer Research \& Nanjing Medical University Affiliated Cancer Hospital, Nanjing, China). \\ Comment on: Mayr R, Fritsche HM, Zeman F, et al. Sarcopenia predicts 90-day mortality and postoperative complications after radical cystectomy for \\ bladder cancer. World J Urol 2018;36:1201-7.
}

Submitted Aug 28, 2018. Accepted for publication Sep 03, 2018.

doi: $10.21037 /$ tau.2018.09.03

View this article at: http://dx.doi.org/10.21037/tau.2018.09.03

Radical cystectomy (RC) with pelvic lymph node dissection, preceded by cisplatin-based neoadjuvant chemotherapy in appropriate patients, remains the gold-standard in the treatment of muscle invasive bladder cancer (MIBC) $(1,2)$. $\mathrm{RC}$ is a complex operation performed in an elderly (and sometimes unhealthy) patient population, with significant complication rates (as high as $60 \%$ ) and perioperative mortality rates $(9 \%)$ reported at 90 -days $(3,4)$.

Unfortunately, RC utilization in the MIBC population is low. In 2010 Gore et al. published on 3,262 patients with MIBC from the SEER-Medicare database and noted that RC utilization was only $21 \%$ in this population (5). Factors contributing to these observations included proximity to specialized medical care, patient comorbidities, and patient age. A subsequent similar analysis of this same dataset dating between 2002 to 2011 showing no progression in RC utilization (19\%) with similar sociodemographic barriers (6). The underlying themes behind underutilization of RC can be broadly summarized as difficulties in either accessing or tolerating care. While issues with access to care reflect U.S. Healthcare System deficiencies that are not often modifiable in the short-term, an individual patient's ability to tolerate surgery may be improved. Thus, there exists a growing interest in identifying modifiable risk factors for post-RC morbidity, mortality, and prolonged recovery. In that regard, sarcopenia, as a potential sign of nutritional insufficiency, frailty, and poor exercise tolerance, is an interesting measure of potentially modifiable patient factors.

The article by Mayr et al. published in World Fournal of Urology, evaluates pre-RC sarcopenia as a predictor of 90-day mortality and morbidity (7). The authors report on 327 patients with MIBC, all of whom had CT imaging of the abdomen within 30 days of surgery. Cross-sectional skeletal muscle surface area at L3 was used to calculate the lumbar skeletal muscle index with comparative evaluation using established gender and body mass index based cutoffs. In this population, 90-day mortality was more likely among sarcopenic patients, even after controlling for American Society of Anesthesiologists (ASA) score, age, and oncologic stage (OR 2.59; 95\% CI: 1.13-5.95; P=0.025). Furthermore, sarcopenia was an independent predictor of complications greater than Clavien-Dindo 3b (OR 2.00; 95\% CI :1.233.26; $\mathrm{P}=0.005$ ) but only trended toward predicting all major complications (Clavien-Dindo $\geq 3$ a).

The current article adds to the existing body of literature on the subject of sarcopenia as a predictor of outcome following RC. Several groups have previously shown that sarcopenia is related to decreased overall survival and cancer-specific survival after RC $(8,9)$. Others have suggested a relationship between pre-operative sarcopenia and length of hospital stay following RC (10). Smith et al. reported an association between sarcopenia and 30-day complication rates, particularly in female patients (11). They were unable to show a statistically significant relationship between sarcopenia and 30-day complications in the overall cohort using total psoas area as a continuous variable, but cutoff points determined via the Youden Index method identified significantly more complications 
in patients meeting the criteria for sarcopenia. Wan et al. demonstrated that skeletal muscle index was significantly associated with major complications (Clavien-Dindo $\geq 3$ ) on multivariate analysis (12). And in 466 patients undergoing RC, Ahmadi et al. showed that total psoas area was an independent predictor of complications at 30- and 90-days in a multivariate model (13).

Preoperative indicators of post-operative outcomes are most useful if they can be modified during the period between the pre-operative assessment and the surgery in question. A Japanese group studying elderly gastrectomy patients demonstrated that sarcopenic patients that participated in a preoperative nutrition and exercise program could increase their grip strength and in a few cases (4/22 patients) became non-sarcopenic (14). Effective initiation of a rehabilitation program for sarcopenic patients prior to RC (so-called pre-habilitation) can be challenging in patients undergoing oncologic surgery due to the time constraints imposed by progressing malignancy. The population of RC patients receiving neoadjuvant chemotherapy may therefore represent a particularly attractive target for pre-habilitation due to the delay in time to surgery necessitated by chemotherapy.

Based on the current paper and the existing literature, we may conclude that pre-operative sarcopenia (which can easily be measured in all patients from axial imaging) is an objective predictor of a patient's ability to tolerate and recover from RC. Furthermore, sarcopenia is an indicator of potentially modifiable issues with nutrition and exercise tolerance. Ideally, sarcopenia measured precystectomy, will identify patients at risk for prolonged or complicated recovery after RC, and trigger the institution of appropriate pre-habilitative measures which may correct the underlying issues. Ongoing clinical trials which further explore this concept will contribute to our understanding of the potential impact of pre-habilitation, and our ability to intervene on at-risk patients prior to cystectomy with the ultimate goal of reduced treatment related morbidity.

\section{Acknowledgements}

None.

\section{Footnote}

Conflicts of Interest: The authors have no conflicts of interest to declare.

\section{References}

1. National Comprehensive Cancer Network. Bladder Cancer (Version 5.2018). Available online: https://www. nccn.org/professionals/physician_gls/pdf/bladder.pdf

2. Chang SS, Bochner BH, Chou R, et al. Treatment of NonMetastatic Muscle-Invasive Bladder Cancer: AUA/ASCO/ ASTRO/SUO Guideline. J Urol 2017;198:552-9.

3. Aziz A, May M, Burger M, et al. Prediction of 90-day mortality after radical cystectomy for bladder cancer in a prospective European multicenter cohort. Eur Urol 2014;66:156-63.

4. Shabsigh A, Korets R, Vora KC, et al. Defining early morbidity of radical cystectomy for patients with bladder cancer using a standardized reporting methodology. Eur Urol 2009;55:164-74.

5. Gore JL, Litwin MS, Lai J, et al. Use of radical cystectomy for patients with invasive bladder cancer. J Natl Cancer Inst 2010;102:802-11.

6. Williams SB, Huo J, Chamie K, et al. Underutilization of Radical Cystectomy Among Patients Diagnosed with Clinical Stage T2 Muscle-invasive Bladder Cancer. Eur Urol Focus 2017;3:258-264.

7. Mayr R, Fritsche HM, Zeman F, et al. Sarcopenia predicts 90-day mortality and postoperative complications after radical cystectomy for bladder cancer. World J Urol 2018;36:1201-7.

8. Psutka SP, Carrasco A, Schmit GD, et al. Sarcopenia in patients with bladder cancer undergoing radical cystectomy: impact on cancer-specific and all-cause mortality. Cancer 2014;120:2910-8.

9. Mayr R, Gierth M, Zeman F, et al. Sarcopenia as a comorbidity-independent predictor of survival following radical cystectomy for bladder cancer. J Cachexia Sarcopenia Muscle 2018;9:505-13.

10. Saitoh-Maeda Y, Kawahara T, Miyoshi Y, et al. A low psoas muscle volume correlates with a longer hospitalization after radical cystectomy. BMC Urol 2017;17:87.

11. Smith AB, Deal AM, Yu H, et al. Sarcopenia as a predictor of complications and survival following radical cystectomy. J Urol 2014;191:1714-20.

12. Wan F, Zhu Y, Gu C, et al. Lower skeletal muscle index and early complications in patients undergoing radical cystectomy for bladder cancer. World J Surg Oncol 2014;12:14.

13. Ahmadi H, Montie JE, Weizer AZ, et al. Patient Psoas 
Muscle Mass as a Predictor of Complications and Survival After Radical Cystectomy. Curr Urol Rep 2015;16:79.

14. Yamamoto K, Nagatsuma Y, Fukuda Y, et al. Effectiveness of a preoperative exercise and nutritional support program for elderly sarcopenic patients with gastric cancer. Gastric Cancer 2017;20:913-8.
Cite this article as: Kaag MG, Raman JD. Sarcopenia is a reliable predictor of outcomes following radical cystectomy for bladder cancer. Transl Androl Urol 2018;7(Suppl 6):S732-S734. doi: 10.21037/tau.2018.09.03 